\title{
Adaptive evolution at mRNA editing sites in soft-bodied cephalopods
}

\author{
Mikhail Moldovan ${ }^{\text {Corresp., }}{ }^{\text {, Zoe Chervontseva }}{ }^{1,2}$, Georgii Bazykin ${ }^{1,2}$, Mikhail S Gelfand ${ }^{1,2}$ \\ ${ }^{1}$ Skolkovo Institute of Science and Technology, Moscow, Russian Federation \\ 2 A.A.Kharkevich Institute for Information Transmission Problems (RAS), Moscow, Russian Federation \\ Corresponding Author: Mikhail Moldovan \\ Email address: mika.moldovan@gmail.com
}

\section{Background}

The bulk of variability in mRNA sequence arises due to mutation - change in DNA sequence which is heritable if it occurs in the germline. However, variation in mRNA can also be achieved by post-

transcriptional modification including mRNA editing, changes in mRNA nucleotide sequence that mimic the effect of mutations. Such modifications are not inherited directly; however, as the processes affecting them are encoded in the genome, they have a heritable component, and therefore can be shaped by selection. In soft-bodied cephalopods, adenine-to-inosine RNA editing is very frequent, and much of it occurs at nonsynonymous sites, affecting the sequence of the encoded protein.

\section{Methods}

We study selection regimes at coleoid A-to-I editing sites, estimate the prevalence of positive selection, and analyze interdependencies between the editing level and contextual characteristics of editing site.

\section{Results}

Here, we show that mRNA editing of individual nonsynonymous sites in cephalopods originates in evolution through substitutions at regions adjacent to these sites. As such substitutions mimic the effect of the substitution at the edited site itself, we hypothesize that they are favored by selection if the inosine is selectively advantageous to adenine at the edited position. Consistent with this hypothesis, we show that edited adenines are more frequently substituted with guanine, an informational analogue of inosine, in the course of evolution than their unedited counterparts, and for heavily edited adenines, these transitions are favored by positive selection. Our study shows that coleoid editing sites may enhance adaptation, which, together with recent observations on Drosophila and human editing sites, points at a general role of RNA editing in the molecular evolution of metazoans. 


\section{Adaptive evolution at mRNA editing sites in soft- 2 bodied cephalopods}

3

$8 \quad 2$ A.A.Kharkevich Institute for Information Transmission Problems (RAS), Bolshoy Karetny Per.

9 19, bld.1, Moscow, Russia 127051

10

11

12

13

14

15

16

17

18
Corresponding Author:

Mikhail A. Moldovan ${ }^{1}$

Bolshoy Boulevard 30, bld. 1, Moscow, 121205, Russia

Email address: mika.moldovan@gmail.com

\section{Abstract}

\section{Background}

The bulk of variability in mRNA sequence arises due to mutation - change in DNA sequence which is heritable if it occurs in the germline. However, variation in mRNA can also be achieved by post-transcriptional modification including mRNA editing, changes in mRNA nucleotide sequence that mimic the effect of mutations. Such modifications are not inherited directly; however, as the processes affecting them are encoded in the genome, they have a heritable component, and therefore can be shaped by selection. In soft-bodied cephalopods, adenine-toinosine RNA editing is very frequent, and much of it occurs at nonsynonymous sites, affecting the sequence of the encoded protein.

\section{Methods}

We study selection regimes at coleoid A-to-I editing sites, estimate the prevalence of positive selection, and analyze interdependencies between the editing level and contextual characteristics of editing site. 


\section{Results}

31 Here, we show that mRNA editing of individual nonsynonymous sites in cephalopods originates

32 in evolution through substitutions at regions adjacent to these sites. As such substitutions mimic

33 the effect of the substitution at the edited site itself, we hypothesize that they are favored by

34 selection if the inosine is selectively advantageous to adenine at the edited position. Consistent

35 with this hypothesis, we show that edited adenines are more frequently substituted with guanine,

36 an informational analogue of inosine, in the course of evolution than their unedited counterparts,

37 and for heavily edited adenines, these transitions are favored by positive selection. Our study

38 shows that coleoid editing sites may enhance adaptation, which, together with recent observations

39 on Drosophila and human editing sites, points at a general role of RNA editing in the molecular

40 evolution of metazoans.

41

42 Introduction

The process of natural selection requires heritable variation to be present in a population and the absence of genetic variants selection could act upon is generally considered to be a factor hampering adaptation (Lush 1937; Smith 1976; Barton and Partridge 2000; Lanfear et al. 2014; Rousselle et al. 2020). Heritable variation is generated mainly by the mutational process (Lewontin 1964; Avery and Hill 1977; Lynch and Walsh 1998). Hence, the mutation rate may be a factor _. affecting the evolution rate, which we, following J. Maynard Smith, define here as the rate of accumulation of beneficial mutations (Smith 1976; Nam et al. 2017; Rousselle et al. 2020). As shown recently, in populations with low genetic variability the mutation rate is indeed correlated with the evolution rate (Rousselle et al. 2020). Thus, in order to adapt, a low-polymorphic population may need additional expressed genetic variability. Here, we test the hypothesis that a potential source of such variability could be introduced by heritable epigenetic modifications, 
54 specifically, mRNA editing (Bass and Weintraub 1988; Gommans et al. 2009; Klironomos et al. 55 2013; Kronholm and Collins 2015).

56

57 58

59

60

61

62

63

64

65

66

67

68

69

70

71

72

73

74

75 76

We consider the A-to-I mRNA editing, where adenine (A) is modified to inosine (I) that is subsequently read by the translation machinery as guanine (G) (Bass and Weintraub 1988). In most of the studied organisms, the A-to-I editing affecting protein sequences is restricted to only a few thousand adenines, with the vast majority of edited adenines located in non-coding regions, e.g. in Alu-repeats (Kim 2004; Ramaswami et al. 2012; Yablonovitch et al. 2017). Edited sites are poorly conserved between species, suggesting that most editing events are non-functional, with a few possible exceptions (Yang et al. 2008; Pinto et al. 2014; Yu et al. 2016). However, in coleoids, soft-bodied cephalopods, about $1 \%$ of adenines in the transcriptome are edited, and re-coding (i.e., affecting the amino acid sequence) and conserved sites comprise considerable fractions (Alon et al. 2015; Liscovitch-Brauer et al. 2017). One explanation for this phenomenon comes from the observation that the conserved editing sites tend to be edited in the nervous tissue, and editing may contribute to the increased plasticity and complexity of the coleoid nervous system and behavior compared to other extant cephalopods (Nautilus) (Albertin et al. 2015; Alon et al. 2015; Liscovitch-Brauer et al. 2017; Eisenberg and Levanon 2018). This hypothesis is supported by analogous observations in other organisms (Pinto et al. 2014; Yu et al. 2016) and, although indirectly, by the finding that the A-to-I RNA editing has emerged approximately at the same time as the nervous systems of multicellular organisms have become more complex (Jin et al. 2009).

A-to-I editing is not absolutely efficient and, if it occurs at a non-synonymous site, would result in two non-identical proteins with a varying ratio (Gommans et al. 2009; Liscovitch-Brauer et al. 2017; Yablonovich et al. 2017). The efficiency of mRNA editing depends on the strength of the site motif and the local mRNA secondary structure (Morse et al. 2002; Reenan 2005; Gommans 
77 et al. 2009; Alon et al. 2012; Savva et al. 2012; Klironomos et al. 2013; Rieder et al. 2013;

78 Liscovitch-Brauer et al. 2017). As the sequence and structure requirements seem to be relatively

79 weak, mRNA editing sites have been proposed to constantly emerge at random points of the 80 genome (Gommans et al. 2009; Xu and Zhang 2014).

To date, four models of A-to-I editing site evolution have been proposed. (i) Most A-to-I editing sites generally are not adaptive and mainly arise at positions with tolerable, i.e. effectively neutral or mildly deleterious, A-to-G substitutions (Xu and Zhang 2014). (ii) A-to-I editing is a mechanism of rescuing deleterious G-to-A substitutions (Jiang and Zhang 2019). (iii) A-to-I editing, generating multiple protein variants, is important for the advantageous transcriptome diversification, and hence the individual sites should be conserved (Liscovitch-Brauer et al. 2017; Eisenberg and Levanon 2018). (iv) The potential of A-to-I editing to mimic A-to-G substitutions is advantageous, and thus A-to-I editing sites function as transitory states when an advantageous mutation has not yet occurred (Popitsch et al., 2020). model (iv) (Popitsch et al., 2020), while editing sites in coleoids are largely considered as means for proteome diversification as in model (iii) (Liscovitch-Brauer et al. 2017; Eisenberg and Levanon 2018) or be selectively neutral (Jiang and Zhang 2019). We attempt to resolve this controversy by detailed analysis of substitution patterns and selection regimes, taking into account the varying strength of A-to-I editing at different sites. benefits of A-to-I editing consistent with model (iv). Indeed, if there is a position in the genome occupied by an adenine, but guanine in this position would yield a fitter genotype, there are two evolutionary pathways for adaptation: through an A-to-G substitution at this site, or through 
100 emergence of a local sequence context yielding or reinforcing A-to-I editing of this site. If the

101 selective benefit conferred by both pathways is comparable, which of them will be taken will

102 depend on the probability of the corresponding mutation (Yampolsky and Stolzfus 2001). A

103 specific mutation is needed in the first scenario; by contrast, many different editing context-

104 improving mutations could yield a fitter genotype, and the waiting time for any such mutation

105 could be shorter (Durrett and Schmidt 2008). As a result, selection would lead to emergence of the 106 adaptive editing phenotype.

107 We propose that non-conserved coleoid A-to-I mRNA editing sites, comprising the larger 108 percentage relative to the conserved ones, could function as substitutes of beneficial A-to-G 109 substitutions in low-polymorphic coleoid populations. We show that the levels of cephalopod A110 to-I editing heavily depend on the sequence of adjacent regions, and hence are influenced by a 111 multitude of possible mutations. Critically, we show that edited adenines are more frequently 112 substituted in related species to guanines and less frequently, to cytosines or thymines, than non113 edited ones. At strongly edited sites, the adenine-to-guanine transitions are favored by positive 114 selection. Our results suggest that, while conserved coleoid editing sites could be functionally 115 important per se, a large subset of non-conserved editing sites could play a role in the adaptive 116 evolution by introducing, at least in a fraction of transcripts, guanines that are beneficial at the 117 given positions. When this study had been completed, a similar observation was made for 118 Drosophila and human editing sites by analysis of genomic polymorphisms (Popitsch et al., 2020).

119 This indicates that A-to-I editing could have similar, important evolutionary roles in multiple 120 metazoan lineages.

121

\section{Materials \& Methods}


Data. Transcriptomes for all six considered species, O. vulgaris, O. bimaculoides, $S$.

124

125

126

127

128

129

130

131

132

133

134

142 143

144

esculenta, L. pealei, N. pompilius, and A. californica, parameters of editing sites, and tables of conserved editing sites were taken from the online supplementary data of Liscovitch-Brauer et al. 2017 (Fig. 1). Genomic read data were downloaded from the SRA database. S. esculenta and $O$. vulgaris genomic read data were taken from bioproject PRJNA299756, L. pealei, from PRJNA255916, and O. bimaculoides, from PRJNA270931.

Annotation of structured and unstructured regions. To estimate the structural potential of each position we used Z-score values obtained by the RNASurface program (Soldatov et al. 2013). Here, Z-score of a sequence is defined as $Z=(E-\mu) / \sigma$ where $E$ is the minimal free energy of a biological sequence, $\mu$ and $\sigma$ are the mean and standard deviation of the energy distribution of shuffled sequences with preserved length and average dinucleotide composition. The program was run with parameters maximal sliding window length 350 and minimal sliding window length 20 . From the RNASurface output, structural potential of overlapping segments was inferred. Each position of each transcript was assigned the best (minimal) Z-score of all structured segments containing it, if it was less than -2, otherwise it was assigned null value. As a result, each transcript was divided into structured and unstructured regions with a $Z$-score value assigned to all positions in the structured regions. The difference between the structural potential upon the A-to-G change (Fig. 2D) was considered if its absolute value exceeded 2.

Analysis of polymorphisms. Genomic reads were mapped onto transcriptomes with bowtie2 (Langmead and Salzberg 2012) using the --sensitive-local run mode. After the sorting of the resulting read alignment files with the samtools package (Li et al. 2009), diploid genotypes were called with bcftools (Narasimhan et al. 2016). Next, we discarded all non-SNP variants and 
145 variants with the quality score below 20 . We computed synonymous nucleotide diversity $\pi_{\mathrm{s}}$ with

146 the pairwise haplotype comparison implemented in the PAML package (Yang 2007).

147 Alignments. To construct multiple transcriptome alignments, we selected a transcriptome

148 of one species and performed BLASTn (Altschul et al. 1990) with the E-value threshold of 10-15

149 against the transcriptomes of the remaining species. Resulting alignment was obtained by merging

150 of the pairwise BLASTn alignments. The results showed only a negligible dependence on the

151 choice of the seed species.

Context analysis. Site LOGOs were built with the WebLOGO server (Crooks 2004). $R$

153

154

155

156

157

158

159

160

161

162

163 as:

164 165

values for mismatches in contexts of non-conserved editing sites were defined as:

$$
R_{N_{1}, N_{2}}^{ \pm 1}=\frac{p\left(\mathrm{E} N_{1}, \mathrm{~A} N_{2}\right)}{p\left(\mathrm{AN}_{1}, \mathrm{~A} N_{2}\right)}
$$

where $N_{1}$ and $N_{2}$ represent nucleotides in positions +1 and -1 relative to the considered adenine, $p\left(\mathrm{EN}_{1}, \mathrm{~A} N_{2}\right)$ is the probability of a mismatch at position +1 or -1 relative to the considered adenine that is edited in one of the two considered species and not edited an another, defined as:

$$
p\left(\mathrm{E} N_{1}, \mathrm{~A} N_{2}\right)=\frac{\#\left(\mathrm{E} N_{1}, \mathrm{~A} N_{2}\right)}{\#(E, A)}
$$

with $\#(E, A)$ and $\#\left(E N_{1}, \mathrm{AN} N_{2}\right)$ being the number of homologous A-E states and the number of contextual $N_{l}-N_{2}$ mismatches associated with the A-E pairs, respectively.

$p\left(\mathrm{~A} N_{l}, \mathrm{~A} N_{2}\right)$ is the respective probability when both homologous adenines are non-edited defined

$$
p\left(\mathrm{AN}_{1}, \mathrm{~A} N_{2}\right)=\frac{\#\left(\mathrm{~A} N_{1}, \mathrm{~A} N_{2}\right)}{\#(A, A)}
$$

$\#(A, A)$ and $\#\left(\mathrm{AN}_{1}, \mathrm{AN} N_{2}\right)$ being the number of homologous $\mathrm{A}-\mathrm{A}$ states and the number of $N_{1}-N_{2}$ mismatches adjacent to the A-A pairs, respectively. 
166 The statistical significance of the $R$ values was assessed by the chi-squared contingency test with

167 the Bonferroni correction on the number of $N_{1}-N_{2}$ mismatch types. A $2 \times 2$ Contingency matrix $S$

168 used in the chi-squared test was constructed from the numbers used to define $p\left(\mathrm{E} N_{l}, \mathrm{~A} N_{2}\right)$ and

$169 p\left(\mathrm{~A} N_{1}, \mathrm{~A} N_{2}\right):$

170

$$
S=\left(\begin{array}{cc}
\#\left(\mathrm{EN}_{1}, \mathrm{AN}_{2}\right) & \#\left(\mathrm{AN}_{1}, \mathrm{AN}_{2}\right) \\
\#(E, A) & \#(A, A)
\end{array}\right)
$$

171

Substitution matrix. For a considered species, we considered its closest relative and an available species, we used maximum parsimony (MP) to reconstruct ancestral states. Thus, for a position in the alignment, the ancestral state of nucleotide $N$ was inferred if the closest relative and an outgroup had the same nucleotide $N^{\text {anc}}$; an ancestral adenine was considered to be edited if the homologous adenines in the closest relative and an outgroup were edited. The substitution matrix was thus comprised of counts inferred by MP, \#( $\left.N^{\text {anc }} \rightarrow N\right)$. expression for the $R$ value. For substitutions at SES:

182

$$
R_{\rightarrow N}^{\mathrm{syn}}=\frac{p\left(\mathrm{E}^{\mathrm{syn}}, N\right)}{p\left(\mathrm{~A}^{\mathrm{syn}}, N\right)}
$$
where $\mathrm{E}^{\mathrm{syn}}$ are synonymous editing sites, i.e. edited adenines that, when substituted to guanine, do

184 not change the amino acid, and, similarly, $\mathrm{A}^{\text {syn }}$ are synonymous unedited adenines. An analogous 185 formula was applied for non-synonymous editing sites. The definitions of probabilities $p\left(\mathrm{E}^{\mathrm{syn}}, N\right)$ 186 and $p\left(\mathrm{~A}^{\mathrm{syn}}, N\right)$ are in this case analogous to those used in the context analysis, see above. 
188 the expression for the $R$ value. For mutations to SES we have:

189

$$
R_{N \rightarrow}^{s y n}=\frac{p^{*}\left(N^{\mathrm{syn}}, \mathrm{E}^{\mathrm{syn}}\right)}{p^{*}\left(N^{\mathrm{syn}}, \mathrm{A}^{\mathrm{syn}}\right)}
$$
substituted with adenine and with guanine would yield the same amino acid. $p^{*}$ are, just like in

192 formula (3), conditional probabilities:

$$
\begin{aligned}
& p^{*}(N, \mathrm{E})=p(N, \mathrm{E}) / \frac{\# \mathrm{E}}{\# \mathrm{E}+\# \mathrm{~A}} \\
& p^{*}(N, \mathrm{~A})=p(N, \mathrm{~A}) / \frac{\# \mathrm{~A}}{\# \mathrm{E}+\# \mathrm{~A}}
\end{aligned}
$$

An analogous formula is applied to NES, with $N^{\text {non }}$ representing nucleotides, that, when 196 substituted with adenine and with guanine would yield different amino acids. substitutions to guanines and to pyrimidines separately by applying the $d N / d S$ measure to edited adenines with a subsequent normalization by $d N / d S$ of unedited adenines. Thus, for substitutions to $\mathrm{G}$ we applied the formula (Suppl. Fig. S9):

$$
\frac{d N(\mathrm{E} \rightarrow \mathrm{G})}{d S(\mathrm{E} \rightarrow \mathrm{G})} / \frac{d N(\mathrm{~A} \rightarrow \mathrm{G})}{d S(\mathrm{~A} \rightarrow \mathrm{G})}
$$

where $d N$ were calculated for all codons and $d S$, for four- and six-fold degenerate codons. An 203 analogous formula was used to estimate selection acting on E-to-Y substitutions. Next, we applied 204 this measure separately for 10\% editing level (EL) bins, counted Pearson's correlation coefficient 205 and applied the $F$ statistic to estimate the significance of the obtained correlation. 

synonymous substitutions were considered for edited adenines, and synonymous, for unedited 208 adenines:

$$
\frac{d N(\mathrm{E} \rightarrow \mathrm{G})}{d S(\mathrm{~A} \rightarrow \mathrm{G})}=\frac{p\left(\mathrm{E}^{\mathrm{non}} \rightarrow \mathrm{G}\right)}{p\left(\mathrm{~A}^{\mathrm{syn}} \rightarrow \mathrm{G}\right)} * \frac{\xi^{\mathrm{non}}}{\xi^{\mathrm{syn}}}
$$

where $\xi^{\text {non }}$ and $\xi^{\text {syn }}$ are normalizing coefficients accounting for differences in codon probabilities and different probabilities of, respectively, synonymous and non-synonymous substitutions under the neutral evolution assumption. These coefficients are defined as:

$$
\xi^{\text {non }}=/\left(\sum_{N_{1} N_{2} N_{3} \in\{\mathrm{A}, \mathrm{T}, \mathrm{G}, \mathrm{C}\}^{3}} f\left(N_{1} N_{2} N_{3}\right) \times K^{\mathrm{non}}\left(N_{1} N_{2} N_{3}, \mathrm{~A} \rightarrow \mathrm{G}\right)\right)
$$$$
\xi^{\text {syn }}=/\left(\sum_{N_{1} N_{2} N_{3} \in\{\mathrm{A}, \mathrm{T}, \mathrm{G}, \mathrm{C}\}^{3}} f\left(N_{1} N_{2} N_{3}\right) \times K^{\mathrm{syn}}\left(N_{1} N_{2} N_{3}, \mathrm{~A} \rightarrow \mathrm{G}\right)\right)
$$

where $f\left(N_{1} N_{2} N_{3}\right)$ is the codon frequency while $K^{\text {non }}$ and $K^{\text {syn }}$ are, respectively, the numbers of possible non-synonymous and synonymous A-to-G substitutions in a given codon. equal to the observed substitution frequencies. For the analysis of parallel evolution, two-tailed 
224 to compare the results for different types of sites, random 100-sequence samples of each type were 225 compared with the Wilcoxon signed-rank test.

226

227 228

229

230

231

232

233

234

235

236

237

238

239

240

241

242

243

244

245

246

247

Data availability. Ad hoc scripts were written in Python. Graphs were built using R. All

scripts and data analysis protocols are available online at https://github.com/mikemoldovan/coleoidRNAediting.

\section{Results}

Editing level is associated with the local and global sequence context. We studied the A-to-I editing using available genomic read libraries, transcriptomes, and editing sites data for four coleoids, closely related octopuses Octopus vulgaris and O. bimaculoides, squid Loligo pealei, and cuttlefish Sepia esculenta (Liscovitch-Brauer et al. 2017). As outgroups, we considered nautiloid Nautilus pompilius and gastropod mollusk Aplysia californica (Liscovitch-Brauer et al. 2017) (Fig. 1A).

The action of editing sites as surrogates of beneficial A-to-G substitutions presumes advantageous enhancement of editing probabilities at individual sites. As A-to-I editing is to be affected by the local sequence context (Alon et al. 2012; Liscovitch-Brauer et al. 2017) and the RNA secondary RNA structure (Morse et al. 2002; Reenan 2005; Gommans et al. 2009; Savva et al. 2012; Klironomos et al. 2013; Rieder et al. 2013), one would expect, firstly, contextual differences around weakly $v s$. heavily edited sites and, secondly, contextual mutations associated with changes in editing status. Indeed, we have observed a previously unnoted dependence of the editing level (EL) (Fig. 1B), defined as the percent of transcripts containing I at the considered site at the moment of sequencing (Fig. 2A, Suppl. Fig. S1), on the site context ( \pm 1 motif). Certain changes in the \pm 1 motif, specifically, an increase in the preference for $\mathrm{G}$ or $\mathrm{T}$ at the +1 position, are associated with the increase of EL, although its information content of the motif remains 
248 approximately the same. Although the \pm 1 motif of both weakly and strongly edited sites is

249 consistent with the ADAR (adenosine deaminases acting on RNA) profile (Alon et al. 2012;

250 Liscovitch-Brauer et al. 2017), this observation could point to the action of different ADAR

251 enzymes or to different modes of action of the same enzyme on strongly and weakly edited sites.

252 There also seem to be some differences between the motifs of conserved and non-conserved sites

253 (Suppl. Fig. S2).

254

The analysis of non-conserved editing sites (NCES) in the octopus pair demonstrates overrepresentation of mismatches in the \pm 1 motif of the edited adenines reinforcing the local

256

257

258

259

260

261

262

263

264

265

266

267

268

269

270

editing context compared to the homologous unedited adenines, for which the editing context is not observed (See Materials and Methods, Suppl. Fig. S3). Thus, both the editing status and the EL of a site are associated with substitutions in the \pm 1 motif. In the squid-cuttlefish pair, the higher number of mutations obscures this analysis.

To estimate the size of the region that affects editing, we have measured the correlation between the editing level difference in conserved editing sites (CES) in closely related species and the number of mismatches in variable-sized windows centered at edited adenines. The window size yielding the largest correlation coefficient shows the average span of the context affecting the ADAR performance. For the Octopus pair, the highest correlation has been obtained at the window size of $\sim 100$ nucleotides (Suppl. Fig. S4), consistent with previous estimates for the length of the region affecting editing (Liscovitch-Brauer et al. 2017).

Editing level is affected by secondary structure in adjacent RNA. The A-to-I editing in model species depends on large RNA structures spanning hundreds of nucleotides in addition to the local sequence context (Morse et al. 2002; Reenan 2005; Ensterö et al. 2009; Rieder et al. 2013; Kurmangaliyev et al. 2015) as the ADAR-mediated mRNA editing generally requires secondary 
271 RNA structures (Gommans et al. 2009; Farajollahi and Maas 2010; Xu and Zhang 2014). Thus,

272 we have assessed the link between RNA secondary structure and ELs of focal sites.

273 We have predicted structured segments in the transcripts of all six considered species. As

274 the fraction of adenines located within structured segments is the same for all cephalopod species, 275 including Nautilus (Suppl. Fig. S5), our secondary structure analyses are not systematically 276 influenced by the GC-content of the studied genomes (Wang et al. 1984). Then we have assessed 277 the contribution of mRNA secondary structure to the editing process by comparing structural 278 contexts of edited and unedited adenines (Materials and Methods). The fraction of edited adenines 279 located in putative structured regions is higher than the respective fraction for non-edited sites. 280 Moreover, sites that are more highly edited (Fig. 2B) as well as sites conserved between more 281 distant species (Fig. 2D) tend to be more structured.

To uncover the connection between the strength of a local secondary RNA structure and 283 the editing status at individual sites, we have compared the fractions of non-conserved editing sites 284 (NCES) located within structured segments in edited vs. non-edited states. We considered the 285 Octopus pair and the squid-cuttlefish pair. For both pairs, we have compared CES and NCES. For 286 NCES in both species pairs we have observed significantly more cases when the edited site in a 287 pair is more structured than the unedited one while the control CES set shows no bias (binomial 288 test $p<10^{-3}$ for all pairs; Fig. 2C, Suppl. Fig. S6).

Not only the fact of editing, but the difference in editing levels is linked to local secondary 290 structures. For the closely related Octopus pair, we have calculated correlations between 291 differences in ELs of homologous edited adenines and differences in their structure Z-scores 292 (Suppl. Fig. S7). Almost no correlation $(r=0.1$, t-test $p<0.05)$ is seen when the EL difference is 293 small $(>5 \%)$, whereas for large differences in ELs $(>50 \%)$ the correlation is substantial $(r=0.7$, t- 
294 test $p<0.05)$. A likely explanation is that larger differences are indeed due to the strength of the

295 local secondary structure, whereas small differences in ELs arise as consequences of random noise.

296 Consistent with the observations above, if we consider structures around edited adenines and their

297 unedited homologs, setting the ELs of unedited adenines to 0, we observe a similar, although a

298 weaker trend (Suppl. Fig. S7), with correlations reaching 0.4 (t-test $p<0.05$ ) when the ELs of

299 NCES are high.

300 The observations about local contexts, both the \pm 1 motif and RNA structures, imply that

301 mutations near editing sites influence the editing status as well as the editing level.

302 Edited adenines are often substituted by guanines. If edited adenines indeed frequently

303 mimic the beneficial guanine state, the substitution patterns of edited and unedited adenines should

304 differ, with edited adenines being more prone to substitutions to guanine and less prone to

305 substitutions to cytosine or thymine (Popitsch et al. 2020). Firstly, we performed the analysis of

306 the species pairs to infer the properties of A-G mismatches at editing sites. For a pair of considered

307 species, we define $R$ as the mismatch probability for an edited adenine divided by the probability

308 of the same mismatch for an unedited adenine: $R_{N}=p(\mathrm{E}, N) / p(\mathrm{~A}, N)$, where $\mathrm{E}$ and $\mathrm{A}$ are, respectively,

309 edited and not edited adenines in one species, and $N$ is the non-E, non-A nucleotide at the

310 homologous site in the other organism. Similar formulas are applied when we consider directed

311 substitutions instead of mismatches. If a pair of the ancestral and the descendant species is

312 considered, we use notation $R_{\rightarrow N}$ to identify the directionality. $R_{\rightarrow N}=p(\mathrm{E} \rightarrow N) / p(\mathrm{~A} \rightarrow N)$, where

$313 p(\mathrm{E} \rightarrow N)$ and $p(\mathrm{~A} \rightarrow N)$ are, respectively, the probabilities of the substitution of the edited and non-

314 edited adenine to $N$. Similarly, notation $R_{N \rightarrow}$ is used when substitutions from ancestral $N$ to $\mathrm{E}$ and

315 A are considered: $R_{N \rightarrow}=p(N \rightarrow \mathrm{E}) / p(N \rightarrow \mathrm{A})$. Higher values of $R_{N \rightarrow \text { imply that the ancestral }}$ 
316 nucleotide $N$ is more likely to be substituted by an edited adenine, compared to an unedited 317 one.

We have observed a striking dependence of the calculated mismatch probabilities on the 319 editing status of the adenines and their ELs. In the Octopus pair, $R_{\mathrm{G}}$ and $R_{\mathrm{Y}}$ (Y denotes pyrimidine, $320 \mathrm{C}$ or $\mathrm{T}$ ) differ both in value and in the dependence on the EL (Fig. $3 \mathrm{AB}$ ). Indeed, $R_{\mathrm{G}}$ is always 321 higher than $R_{\mathrm{Y}}$ with $R_{\mathrm{G}}$ further increasing and $R_{\mathrm{Y}}$ decreasing as the EL increases. The probability 322 for an adenine to be substituted by a guanine in the $O$. vulgaris lineage is $\sim 8$ times higher when 323 the homologous adenine is strongly edited in O. bimaculoides than when it is not (Fig. 3A). For 324 the more distantly related squid-cuttlefish pair, we observe a similar although less pronounced 325 effect. For all distant pairs, that is, Octopus-squid/cuttlefish, $R_{\mathrm{G}}$ shows no or only a weak 326 dependence on the EL.

We have calculated $R$ values separately for non-synonymous editing sites (NES), which comprise between $64.6 \%$ and $65.7 \%$ of all detected coleoid editing sites, and for synonymous editing sites (SESs) which comprise the remaining 34.3\% to 35.4\%. NES (Suppl. Fig. S8AB) 330 demonstrate the same pattern as described above for all sites, whereas for SES, we see no 331 dependence of $R_{\mathrm{Y}}$ on the EL (Suppl. Fig. S8CD). NES demonstrate very low $R_{\mathrm{Y}}$ at high ELs. These 332 patterns suggest that at highly edited nonsynonymous adenine sites, any nucleotide other than 333 guanines are impeded by strong negative selection; whereas the guanine states at such sites are 334 frequent. Thus, at non-synonymous NCES, the selection patterns differ from those at non335 conserved adenines: Y mismatches with NCES experience stronger negative selection than $\mathrm{Y}$ 336 mismatches with non-edited adenines, and stronger positive and/or weaker negative selection 337 acting on E-to-G or G-to-E substitutions compared to A-to-G or G-to-A ones, respectively. 

selection at edited sites, we have calculated the $d N / d S$ ratios separately for mismatches of edited and unedited adenines with guanines and with pyrimidines (Suppl. Fig. S9). For weakly edited adenines, the $d N / d S$ values of mismatches with guanines and with pyrimidines are approximately substitutions to guanine is two- to threefold higher, compared to unedited adenines, while the respective ratio for pyrimidines is twofold lower. Thus, strongly edited sites evolve under weaker purifying selection against E-to-G and/or G-to-E transitions and stronger purifying selection against E-to-Y and/or Y-to-E substitutions.

To distinguish between positive selection and relaxation of negative selection at these sites,

348

we have calculated $d N / d S$ for A-G mismatches where $d N$ and $d S$ are calculated for edited and unedited adenines, respectively. It is larger than 1 at high ELs for the closely related octopus species pair (Fig. 4), indicating positive selection acting on the E-to-G transition: heavily edited adenines are positively selected for substitutions to guanine.

E-to-G substitutions versus G-to-E substitutions. In theory, two processes could lead to the increase in the observed $R$ and $d N / d S$ values of edited sites - the increased frequency of either E-to-G or G-to-E substitutions. To distinguish between these possibilities, we use the procedure described in Materials and Methods to calculate the frequencies of all types of substitutions for each species since its closest ancestor. We also consider the more robust, averaged substitution frequencies for the Octopus pair and for the squid-cuttlefish pair. As the frequencies of substitutions to edited and non-edited adenines are calculated separately, we introduce the normalized, directional measure $Q_{\rightarrow *}$ reflecting the preference of edited adenines to substitute to guanine: 
361

$$
Q_{\rightarrow *}=\frac{R_{\rightarrow \mathrm{G}}}{R_{\rightarrow \mathrm{Y}}}=\frac{p(\mathrm{E} \rightarrow \mathrm{G})}{p(\mathrm{~A} \rightarrow \mathrm{G})} / \frac{p(\mathrm{E} \rightarrow \mathrm{Y})}{p(\mathrm{~A} \rightarrow \mathrm{Y})}=\frac{p(\mathrm{E} \rightarrow \mathrm{G})}{p(\mathrm{E} \rightarrow \mathrm{Y})} / \frac{p(\mathrm{~A} \rightarrow \mathrm{G})}{p(\mathrm{~A} \rightarrow \mathrm{Y})}
$$

362 By this definition, the $Q_{\rightarrow *}$ measure is an indicator of the joint effect of the prevalence of E-to-G

363 over A-to-G substitutions and of the underrepresentation of E-to-Y relative to A-to-Y substitutions.

364 For the squid-cuttlefish clade, and for SESs and NESs considered separately, $Q_{\rightarrow *}$ ranges from

3653.49 to 6.4 (Fig. 3C, Suppl. Fig. S10AB), in all cases being significantly higher than 1 expected

366 under a neutral model $(p<0.005)$. Hence, as in the case of pairwise comparison of extant species

367 (Fig. 3AB), edited adenines have a substitution pattern strikingly different from that of unedited

368 adenines, and are likely to mutate into guanines.

369 However, large values of $Q_{\rightarrow *}$ may be explained by two effects, high $R_{\rightarrow \mathrm{G}}$ of E-to-G

370 substitutions or low $R_{\rightarrow \mathrm{Y}}$ of E-to-Y substitutions (Fig. 3C) both yielding $R_{\rightarrow \mathrm{G}}$ higher than $R_{\rightarrow \mathrm{Y}}$.

$371 R_{\rightarrow \mathrm{G}}$ is higher than $1(p<0.005)$, thus indicating that an edited adenine is more likely to be

372 substituted by guanine than an unedited adenine. Combined with $R_{\rightarrow \mathrm{Y}}$ being smaller than 1

$373(p<0.005)$, this indicates that in fact both effects contribute to the observed $Q_{\rightarrow} *$ values. A similar

374 pattern holds if we consider NES and SES separately: $R_{\rightarrow \mathrm{G}}$ is higher than $R_{\rightarrow \mathrm{Y}}$, although for NES

375 high $Q_{\rightarrow *}$ can be almost entirely attributed to $R_{\rightarrow \mathrm{G}}$, and for SES, to $R_{\rightarrow \mathrm{Y}}(p<0.005)$ (Suppl. Fig.

$376 \mathrm{~S} 10 \mathrm{AB})$.

377 To analyze the directionality of the mutation process that affects editing states, we consider

378 a similar function measuring the degree of prevalence of G-to-E substitutions:

379

$$
Q_{* \rightarrow}=\frac{R_{\mathrm{G} \rightarrow}}{R_{\mathrm{Y} \rightarrow}}=\frac{p^{*}(\mathrm{G} \rightarrow \mathrm{E})}{p^{*}(\mathrm{G} \rightarrow \mathrm{A})} / \frac{p^{*}(\mathrm{Y} \rightarrow \mathrm{E})}{p^{*}(\mathrm{Y} \rightarrow \mathrm{A})}=\frac{p^{*}(\mathrm{G} \rightarrow \mathrm{E})}{p^{*}(\mathrm{Y} \rightarrow \mathrm{E})} / \frac{p^{*}(\mathrm{G} \rightarrow \mathrm{A})}{p^{*}(\mathrm{Y} \rightarrow \mathrm{A})}
$$


380 where probabilities $p^{*}$ are conditional probabilities of a nucleotide mutating to either edited or 381 unedited adenine after taking into account differences in the $\mathrm{E}$ and $\mathrm{A}$ densities in the 382 transcriptomes:

$$
\begin{aligned}
& p^{*}(N \rightarrow \mathrm{E})=p(N \rightarrow \mathrm{E}) / \frac{\# \mathrm{E}}{\# \mathrm{E}+\# \mathrm{~A}} \\
& p^{*}(N \rightarrow \mathrm{A})={ }^{p(N \rightarrow \mathrm{A})} / \frac{\# \mathrm{~A}}{\# \mathrm{E}+\# \mathrm{~A}}
\end{aligned}
$$

For both the Octopus pair and the squid-cuttlefish pair, $Q_{* \rightarrow}$ is larger than $1(p<0.005)$ (Fig.

3c, Suppl. Fig. S10), thus suggesting that guanines tend to be substituted by edited rather than unedited adenines. However, this effect is on average twofold smaller than that for substitutions

388

389

390

391

392

393

394

395 396 397 398 substitutions rather than rescuing deleterious G-to-A substitutions.
401

\section{Discussion}


404

405

406

407

408

409

410

411

412

413

414

415

416

417

418

419

420

421

422

423

424

425

our observations. Editing in coleoids is essential for transcriptome diversification, and results in a more complex phenotype (Liscovitch-Brauer et al. 2017; Eisenberg and Levanon 2018). Indeed, a considerable fraction of coleoid editing sites are conserved between even distantly related species, and a majority of heavily edited sites affect protein sequence (Liscovitch-Brauer et al. 2017). We propose that non-conserved coleoid editing sites could facilitate adaptation by extending selection to regions affecting editing if guanine is the beneficial variant at the editing site. This hypothesis is directly supported by our observations. Indeed, strong dependence of editing on the local context allows for selection of mutations in the vicinity of the editing site, hence extending the variety of beneficial mutations. On the other hand, edited adenines indeed tend to be substituted by guanines, and guanines are selected for if the editing levels of homologous adenines is high. This positive selection pattern is specific to guanine variants, as substitutions of edited adenines to cytosine or thymine are avoided.

An indirect observation also supports our hypothesis. Sizes of the effects such as the Eto-G substitution rate or the rate of positive selection on the guanine variant at editing sites are larger for heavily edited adenines compared to medium and weakly edited ones. This effect could be explained by beneficial A-to-G substitutions provoking selection on adjacent regions, which leads to the increased ELs and hence to the enhanced presence of the guanine-like variant. Indeed, if $\mathrm{G}$ is beneficial at a given site, it would manifest as both positive selection towards $\mathrm{G}$ at this site, and by mutations at adjacent sites yielding higher A-to-I editing level, and hence these two types of effects would be correlated.

Positive selection in favor of E-to-G substitutions. Why would substitutions that recapitulate editing be adaptive? Conceivably, it could be that variability at the transcriptome level 
426 is advantageous by itself, contributing to the proteome diversity, similar to alternative splicing, 427 alternative transcription and translation starts, etc (Raj and van Oudenaarden 2008; Gommans et 428 al. 2009; Pickrell et al. 2010). However, this scenario does not explain positive selection of 429 substitutions to $\mathrm{G}$.

430 Alternatively, editing might create an unconditionally beneficial variant, so that at an edited 431 site, $\mathrm{G}$ is always better than A. Under this scenario, editing could recreate the $\mathrm{G}$ allele previously 432 lost due to a deleterious G-to-A mutation, or produce a novel G variant which is favored by 433 selection but has not been present at this site previously (Jiang and Zhang 2019). This scenario is 434 supported by the observed selection favoring guanines at edited sites.

But why would selection in favor of $\mathrm{G}$ result in an increased A-to-I editing of a fraction of

436 the transcripts, when a "direct" A-to-G genomic mutation at this site would lead to the same result 437 in $100 \%$ of transcripts? One reason could be that mutations creating editing sites and/or increasing 438 editing level are more numerous, and therefore more readily available. For a strongly advantageous 439 mutation (with $4 N_{\mathrm{e} S}>>1$ ) that does not preexist in the population, the time till its fixation equals $4401 /\left(4 N_{\mathrm{e}} s \mu\right)$, where $N_{\mathrm{e}}$ is the effective population size, $s$ is selection in favor of the new mutation, 441 and $\mu$ is the mutation rate, see eq. 3.22 in Kimura 1983. If two types of mutations can yield the 442 desired phenotype, which of them would be the first to fix in an evolving lineage is determined by 443 the product of the corresponding selection and mutation rates.

444 Let $\mu_{1}$ be the rate of the direct mutation, and $s_{1}$, selection in its favor. Assume that an 445 increase in the number of favored transcripts can also be achieved by $M$ other mutations, each 446 characterized by rate $\mu_{2}$ and selection $s_{2}$. The probability that the editing-enhancing mutation will 447 be the first to occur then equals $M \mu_{2} s_{2} /\left(\mu_{1} s_{1}+M \mu_{2} s_{2}\right)$ (Yampolsky and Stolzfus 2001). If $448 M \mu_{2} s_{2}>\mu_{1} s_{1}$, the editing-increasing mutation will typically fix earlier than the direct mutation. As 
449 we show, many tens of sites may affect editing, making $M$ large, and this scenario likely. For 450 example, if the direct A-to-G substitution confers a $10 \%$ increase in fitness, but a $1 \%$ increase can 451 be achieved by changes in editing by mutations at each of 20 other sites, then the editing-increasing 452 change will be the first to occur with probability $2 / 3$ if the mutation rates are uniform. This reasoning only applies if the within-species variability level $N_{e} \mu$ is low $(<<1)$;

454 455

456 457 458 459 460 461 462 463 464 465 466 467 468 469 470 471

otherwise each site will carry a preexisting mutation, and the mutation rate will be less relevant (McCandlish and Stoltzfus 2014). Low variability is indeed a characteristic trait of the considered coleoid species, with synonymous-site pairwise divergence of $2.5 \times 10^{-3}$ for $O$. bimaculoides, $2.2 \times 10^{-3}$ for O. vulgaris, $1.8 \times 10^{-3}$ for S. eculenta, and $4.5 \times 10^{-3}$ for L. pealei (see Materials and Methods). These values imply $N_{e} \mu<<1$, suggesting that evolution can be indeed mutation-limited in this group of species. Low values of $N_{e} \mu$ characteristic of higher animals have been proposed to underlie many aspects of genomic complexity (Lynch 2007); they may also cause the high prevalence of RNA editing in coleoids.

When this study had been completed, Popitsch et al. published a population-genetic study of Drosophila and human A-to-I RNA editing sites, in which they showed a similar pattern of selection at editing sites, with the derived $\mathrm{G}$ state selected upon, whereas $\mathrm{C}$ and $\mathrm{T}$ variants being suppressed, indicating enhanced negative selection (Popitsch et al. 2020). That study indirectly supports our claim about coleoid A-to-I editing sites mimicking beneficial A-to-G substitutions. Furthermore, as coleoids possess many more conserved re-coding A-to-I editing sites than any other studied metazoan group, one might expect the bulk of coleoid editing, especially at heavily edited sites, to be important per se, e.g. for transcriptome diversification, which would result in suppression of any non-adenine variants in editing sites. On the contrary, we have observed positive selection in A-G mismatches, when adenines are heavily edited, with selection acting 
472 specifically on A-to-G transitions. Also, like Popitsch et al., we have observed enhanced negative

473 selection against A-to-C and A-to-T substitutions and mismatches at coleoid editing sites. The

474 consistency of results obtained for coleoids, Drosophila, and human points towards a general role

475 of A-to-I editing sites as imitations and precursors of A-to-G transitions in the evolution of 476 metazoans with low-polymorphic populations.

477 Conservation and function of editing. Earlier, it has been proposed that most editing sites 478 result from tolerable promiscuous ADAR action (Xu and Zhang 2014). However, the A-to-I 479 editing sites in coleoids are under positive selection if ELs are high (Fig. 4). Hence large ELs 480 cannot result simply from the tolerance towards substitutions to guanines at these sites. et al. 2015; Alon et al. 2015; Liscovitch-Brauer et al. 2017; Eisenberg and Levanon 2018; Jiang 483 and Zhang 2019). However, this applies only to conserved, and hence functional, editing sites. We propose that coleoid editing sites form two populations with different properties. Firstly, there are functional editing sites, which are important per se due to their ability to diversify protein products in various tissues and environmental conditions (Savva et al. 2012; Alon et al. 2015; Harjanto et al. 2016; Buchumenski et al. 2017; Duan et al. 2017; Liscovitch-Brauer et al. 2017; Tan et al. 2017). As such sites should be retained over long periods of time, we may consider conservation as a good proxy for functionality. Conserved sites are surrounded by conserved regions 490 (Liscovitch-Brauer et al. 2017), their ELs show dependence on the number of substitutions in 491 adjacent regions (Suppl. Fig. S4), and they comprise up to about a half of A-to-I editing sites in a 492 coleoid transcriptome (Liscovitch-Brauer et al. 2017). 
495 young functional sites should be minimal (Gommans et al. 2009), the general properties of non496 conserved sites should reasonably well represent those of non-functional ones. Non-conserved 497 sites are not flanked by conserved regions, their ELs show no correlation with the number of 498 substitutions in adjacent regions, and their sequence contexts differ from those of the conserved 499 ones (Suppl. Fig. S2). Our hypothesis that (non-conserved) editing sites have an intrinsic 500 evolutionary value does not contradict the fact that some (possibly large) subset of editing sites 501 are functional as editing sites per se from the physiological point of view.

502 Theoretically, our results could have been influenced by underprediction of editing sites.

503 As the mean EL is about 5\%, a site might be easily missed especially in transcripts with low 504 expression levels (Bahn et al. 2011; Alon et al. 2012; Liscovitch-Brauer et al. 2017). However, the 505 majority of our observations are obtained for highly edited adenines, which are predicted with 506 greater accuracy (Bahn et al. 2011), and hence should not be influenced by missing weakly edited 507 sites.

508

Theoretical frameworks and alternative explanations. Our results could be interpreted 509 within several paradigms. Firstly, as noted above, the observations could mean that editing rescues 510 deleterious G-to-A substitutions (Jiang and Zhang 2019). However, as also mentioned above, the 511 estimates of $Q$ values, which represent the mutation process directionality, indicate that E-to-G 512 substitutions differ in terms of the transition/transversion rate from A-to-G ones to a much greater 513 extent, than G-to-E substitutions differ from G-to-A $\left(Q_{\rightarrow^{*}}>>Q_{*^{*}},\right)$; in addition, $Q_{*^{*}}<1$ at non514 synonymous sites (Fig. 3C), again supporting the idea that the E-to-G transitions contribute to the 515 observed effects to a larger degree. Ultimately, this issue would be resolved when more data are 516 available, allowing for the reconstruction of ancestral states of NCES. 
518 (Waddington 1953a, 1953b; Lynch and Walsh 1998; Crispo 2007; Ghalambor et al. 2007;

519 Ghalambor et al. 2015; Levis and Pfennig 2016; Ho and Zhang 2018; Levis and Pfennig 2019).

520 Editing could buffer coleoids against environmental changes — under novel conditions the

521 phenotype changes (adenine is edited and read as guanine), and subsequently this change is

522 reinforced on the genome level by the selection process, which we observe as positive selection

523 pressure on E-to-G transitions. However, at present we have no data on environmental variance in

524 the coleoid A-to-I editing.

525 The preadaptation paradigm refers to a pre-existing structure that has changed its function 526 or acquired a new one in the course of evolution (Darwin 1872; Gould and Vrba 1982; McLennan 527 2008; Ardila 2016; Casinos 2017; Cadotte et al. 2018). Here, as non-functional editing should be 528 mostly effectively neutral (Gommans et al. 2009), it might generate a pool of variants, some of 529 which may become advantageous in the future, when the genetic background or environmental 530 conditions change. However, to claim preadaptation one should determine the function of each 531 editing site, which is not feasible.

532 Hence, the most reasonable framework for our findings seems to be in terms of non533 functional editing sites enhancing the expressed genetic variability, thus contributing to the 534 acceleration of the evolutionary process at sites with beneficial A-to-G substitution. The 535 Continuous Probing Hypothesis (Gommans et al. 2009) states that editing sites, due to the lack of 536 a strict context, constantly emerge at random points of the transcribed genomic regions. Hence, an 537 adenine with a beneficial substitution to guanine could become edited if the editing context 538 emerges around it purely by chance. The context can be further selected upon, resulting in the 
539 mimicking of the beneficial guanine variant. (An extended version of this discussion is provided 540 as Suppl. Mat. 3)

542 as transcription and splicing. These processes depend on regulatory sites and contexts that change

543 the quantity, dynamics (developmental stage, tissue-specificity, response to external conditions),

544 and sequence of encoded proteins and hence are subject to selection (Raj and van Oudenaarden

545 2008; Pickrell et al. 2010). Hence a natural extension of this study would be to systematically

546 assess the evolutionary advantage of noise in information transmission processes in low-

547 polymorphic populations.

548

\section{Conclusions}

550

RNA editing sites are much more numerous in soft-bodied cephalopods (coleoids) than in

551

552

553

554

555

556

557

558

559

560

561

coleoid RNA editing sites to function as surrogates for beneficial A-to-G substitutions. This effect is more pronounced for heavily edited sites. At that, the latter are surrounded by stronger local RNA secondary structure (expectedly) and feature different sequence context (unexpectedly). The RNA structure is even stronger around edited adenines homologous to guanines in sister species. Edited adenines tend to be substituted to guanines, and this tendency is supported by positive selection at highly edited sites.

These observations may be explained by the beneficial effect of increased phenotypic diversity in a low-polymorphic population, enhancing adaptation and facilitating the evolutionary process. Besides, A-to-I editing at sites where $\mathrm{G}$ would be preferred provides a larger (than a single nucleotide position) target for mutations increasing the editing level. Together with similar recent 
562 observations on Drosophila and human editing sites (Popitsch et al. 2020), this points at a general

563 role of RNA editing in the molecular evolution of metazoans.

564

565 Acknowledgements

566

The authors are grateful to Stepan Denisov, Sofya Garushyants, Fyodor Kondrashov, and

567 Eugene Koonin for discussions, criticisms, and suggestions.

568

569

\section{References}

570 Albertin CB, Simakov O, Mitros T, Wang ZY, Pungor JR, Edsinger-Gonzales E, Brenner S, 571 Ragsdale CW, Rokhsar DS. The octopus genome and the evolution of cephalopod neural and 572 morphological novelties. Nature 2015; 524, 220-224; doi:10.1038/nature14668.

573 Alon S, Garrett SC, Levanon EY, Olson S, Graveley BR, Rosenthal JJC, Eisenberg E. The majority 574 of transcripts in the squid nervous system are extensively recoded by A-to-I RNA editing. eLife. 575 2015; 4; doi:10.7554/elife.05198.

576 Alon S, Mor E, Vigneault F, Church GM, Locatelli F, Galeano F, Gallo A, Shomron N, Eisenberg

577 E. Systematic identification of edited microRNAs in the human brain. Genome Research. 2012; 578 22, 1533-1540; doi:10.1101/gr.131573.111.

579 Altschul SF, Gish W, Miller W, Myers EW, Lipman DJ. Basic local alignment search tool. Journal 580 of Molecular Biology. 1990; 215, 403-410; doi:10.1016/s0022-2836(05)80360-2.

581 Ardila A. The Evolutionary Concept of "Preadaptation" Applied to Cognitive Neurosciences. 582 Frontiers in Neuroscience. 2016; 10; doi:10.3389/fnins.2016.00103.

583 Avery PJ, Hill WG. Variability in genetic parameters among small populations. Genetical 584 Research. 1977; 29, 193-213; doi:10.1017/s0016672300017286. 
585 Bahn JH, Lee J-H, Li G, Greer C, Peng G, Xiao X. Accurate identification of A-to-I RNA editing 586 in human by transcriptome sequencing. Genome Research. 2011; 22, 142-150; 587 doi:10.1101/gr.124107.111.

588 Barton N, Partridge L. Limits to natural selection. BioEssays. 2000; 22, 1075-1084; 589 doi:10.1002/1521-1878(200012)22:12<1075::aid-bies5>3.0.co;2-m.

590 Bass BL, Weintraub H. An unwinding activity that covalently modifies its double-stranded RNA 591 substrate. Cell. 1988; 55, 1089-1098; doi:10.1016/0092-8674(88)90253-x.

592 Buchumenski I, Bartok O, Ashwal-Fluss R, Pandey V, Porath HT, Levanon EY, Kadener S. 593 Dynamic hyper-editing underlies temperature adaptation in Drosophila. PLOS Genetics. 2017; 13, 594 e1006931; doi:10.1371/journal.pgen.1006931.

595 Cadotte MW, Campbell SE, Li S, Sodhi DS, Mandrak NE. Preadaptation and Naturalization of 596 Nonnative Species: Darwin's Two Fundamental Insights into Species Invasion. Annual Review of 597 Plant Biology. 2018; 69, 661-684; doi:10.1146/annurev-arplant-042817-040339.

598 Casinos A. From Cuénot's préadaptation to Gould and Vrba’s exaptation: a review. Biological 599 Journal of the Linnean Society. 2017; 121, 239-247; doi:10.1093/biolinnean/blw038.

600 Crispo E. The Baldwin Effect and Genetic Assimilation: Revisiting two Mechanisms of 601 Evolutionary Change Mediated by Phenotypic Plasticity. Evolution. 2007; 61, 2469-2479; 602 doi:10.1111/j.1558-5646.2007.00203.x.

603 Crooks GE. WebLogo: A Sequence Logo Generator. Genome Research. 2004; 14, 1188-1190; 604 doi:10.1101/gr.849004.

605 Darwin, CR. The origin of species by means of natural selection, or the preservation of favoured 606 races in the struggle for life, 6th edition; with additions and corrections. London: John Murray. $607 \quad 1872$. 
608 Duan Y, Dou S, Luo S, Zhang H, Lu J. Adaptation of A-to-I RNA editing in Drosophila. PLOS 609 Genetics. 2017; 13, e1006648; doi:10.1371/journal.pgen.1006648.

610 Durrett R, Schmidt D. Waiting for Two Mutations: With Applications to Regulatory Sequence 611 Evolution and the Limits of Darwinian Evolution. Genetics. 2008; 180, 1501-1509; 612 doi:10.1534/genetics.107.082610.

613 Eisenberg E, Levanon EY. A-to-I RNA editing — immune protector and transcriptome diversifier. 614 Nature Reviews Genetics. 2018; 19, 473-490; doi:10.1038/s41576-018-0006-1.

615 Ensterö M, Daniel C, Wahlstedt H, Major F, Öhman M. Recognition and coupling of A-to-I edited 616 sites are determined by the tertiary structure of the RNA. Nucleic Acids Research. 2009; 37, 6916617 6926; doi:10.1093/nar/gkp731.

618 Farajollahi S, Maas S. Molecular diversity through RNA editing: a balancing act. Trends in 619 Genetics. 2010; 26, 221-230; doi:10.1016/j.tig.2010.02.001.

620 Ghalambor CK, Hoke KL, Ruell EW, Fischer EK, Reznick DN, Hughes KA. Non-adaptive 621 plasticity potentiates rapid adaptive evolution of gene expression in nature. Nature; 2015; 525, 622 372-375; doi:10.1038/nature15256.

623 Ghalambor CK, McKay JK, Carroll SP, Reznick DN. Adaptive versus non-adaptive phenotypic 624 plasticity and the potential for contemporary adaptation in new environments. Functional Ecology. 625 2007; 21, 394-407; doi:10.1111/j.1365-2435.2007.01283.x.

626 Gommans WM, Mullen SP, Maas S. RNA editing: a driving force for adaptive evolution? 627 BioEssays. 2009; 31, 1137-1145; doi:10.1002/bies.200900045.

628 Gould SJ, Vrba ES. Exaptation - a Missing Term in the Science of Form. Paleobiology. 1982; 8, 629 4-15; doi:10.1017/s0094837300004310. 
630 Harjanto D, Papamarkou T, Oates CJ, Rayon-Estrada V, Papavasiliou FN, Papavasiliou A. RNA 631 editing generates cellular subsets with diverse sequence within populations. Nature 632 Communications. 2016; 7; doi:10.1038/ncomms 12145.

633 Hedges SB, Dudley J, Kumar S. TimeTree: a public knowledge-base of divergence times among 634 organisms. Bioinformatics. 2006; 22, 2971-2972; doi:10.1093/bioinformatics/btl505.

635 Ho W-C, Zhang J. Evolutionary adaptations to new environments generally reverse plastic 636 phenotypic changes. Nature Communications. 2018; 9; doi:10.1038/s41467-017-02724-5.

637 Jiang D, Zhang J. The preponderance of nonsynonymous A-to-I RNA editing in coleoids is 638 nonadaptive. Nature Communications. 2019; 10; doi:10.1038/s41467-019-13275-2.

639 Jin Y, Zhang W, Li Q. Origins and evolution of ADAR-mediated RNA editing. IUBMB Life. 640 2009; 61, 572-578; doi:10.1002/iub.207.

641 Kim DDY. Widespread RNA Editing of Embedded Alu Elements in the Human Transcriptome.

642 Genome Research. 2004; 14, 1719-1725; doi:10.1101/gr.2855504.

643 Kimura M. The Neutral Theory of Molecular Evolution. Cambridge University Press. 1983.

644 Klironomos FD, Berg J, Collins S. How epigenetic mutations can affect genetic evolution: Model 645 and mechanism. BioEssays. 2013; 35, 571-578; doi:10.1002/bies.201200169.

646 Kronholm I, Collins S. Epigenetic mutations can both help and hinder adaptive evolution.

647 Molecular Ecology. 2015; 25, 1856-1868; doi:10.1111/mec.13296.

648 Kurmangaliyev YZ, Ali S, Nuzhdin SV. Genetic Determinants of RNA Editing Levels of ADAR 649 Targets in Drosophila melanogaster. Genes|Genomes|Genetics. 2015; 6, 391-396; 650 doi:10.1534/g3.115.024471.

651 Lanfear R, Kokko H, Eyre-Walker A. Population size and the rate of evolution. Trends in Ecology 652 \& Evolution. 2014; 29, 33-41; doi:10.1016/j.tree.2013.09.009. 
653 Langmead B, Salzberg SL. Fast gapped-read alignment with Bowtie 2. Nature Methods. 2012; 9,

654 357-359; doi:10.1038/nmeth.1923.

655 Levis NA, Pfennig DW. Evaluating "Plasticity-First" Evolution in Nature: Key Criteria and 656 Empirical Approaches. Trends in Ecology \& Evolution. 2016; 31, 563-574; 657 doi:10.1016/j.tree.2016.03.012.

658 Levis NA, Pfennig DW. Phenotypic plasticity, canalization, and the origins of novelty: Evidence 659 and mechanisms from amphibians. Seminars in Cell \& Developmental Biology. 2019; 88, 80-90; 660 doi:10.1016/j.semcdb.2018.01.012.

661 Lewontin RC. The Interaction of Selection and Linkage. General Considerations; Heterotic 662 Models. Genetics. 1964; 49(1):49-67.

663 Li, H., Handsaker, B., Wysoker, A., Fennell, T., Ruan, J., Homer, N., Marth, G., Abecasis, G., \& 664 Durbin, R. The Sequence Alignment/Map format and SAMtools. Bioinformatics. 2009; 25, 2078665 2079; doi:10.1093/bioinformatics/btp352.

666 Liscovitch-Brauer, N., Alon, S., Porath, H. T., Elstein, B., Unger, R., Ziv, T., Admon, A., Levanon, 667 E. Y., Rosenthal, J. J. C., \& Eisenberg, E.. Trade-off between Transcriptome Plasticity and 668 Genome Evolution in Cephalopods. Cell. 2017; 169, 191-202.e11; 669 doi:10.1016/j.cell.2017.03.025.

670 Lush, JL. Animal Breeding Plans. Ames, Iowa: Iowa State Press. 1937.

671 Lynch M. The Origins of Genome Architecture. Sinauer Associates. 2007.

672 Lynch, M. \& Walsh, B. Genetics and Analysis of Quantitative Traits, 1st edn. Sinauer, Sunderland, 673 MA. 1998.

674 McCandlish DM, Stoltzfus A. Modeling Evolution Using the Probability of Fixation: History and 675 Implications. The Quarterly Review of Biology. 2014; 89, 225-252; doi:10.1086/677571. 
676 McLennan DA. The Concept of Co-option: Why Evolution Often Looks Miraculous. Evolution:

677 Education and Outreach. 2008; 1, 247-258; doi:10.1007/s12052-008-0053-8.

678 Morse DP, Aruscavage PJ, Bass BL. RNA hairpins in noncoding regions of human brain and 679 Caenorhabditis elegans mRNA are edited by adenosine deaminases that act on RNA. Proceedings 680 of the National Academy of Sciences. 2002; 99, 7906-7911; doi:10.1073/pnas.112704299.

681 Nam K, Munch K, Mailund T, Nater A, Greminger MP, Krützen M, Marquès-Bonet T, Schierup $682 \mathrm{MH}$. Evidence that the rate of strong selective sweeps increases with population size in the great 683 apes. Proceedings of the National Academy of Sciences. 2017; 114, 1613-1618; 684 doi:10.1073/pnas.1605660114.

685 Narasimhan V, Danecek P, Scally A, Xue Y, Tyler-Smith C, Durbin R. BCFtools/RoH: a hidden 686 Markov model approach for detecting autozygosity from next-generation sequencing data. 687 Bioinformatics. 2016; 32, 1749-1751; doi:10.1093/bioinformatics/btw044.

688 Pickrell JK, Pai AA, Gilad Y, Pritchard JK. Noisy Splicing Drives mRNA Isoform Diversity in 689 Human Cells. PLoS Genetics. 2010; 6, e1001236; doi:10.1371/journal.pgen.1001236.

690 Pinto Y, Cohen HY, Levanon EY. Mammalian conserved ADAR targets comprise only a small 691 fragment of the human editosome. Genome Biology. 2014; 15, R5; doi:10.1186/gb-2014-15-1-r5. 692 Popitsch N, Huber CD, Buchumenski I, Eisenberg E, Jantsch M, von Haeseler A, Gallach M. A693 to-I RNA Editing Uncovers Hidden Signals of Adaptive Genome Evolution in Animals. Genome 694 Biology and Evolution. 2020; 12:345-357; doi:10.1093/gbe/evaa046.

695 Raj A, van Oudenaarden A. Nature, Nurture, or Chance: Stochastic Gene Expression and Its 696 Consequences. Cell. 2008; 135, 216-226; doi:10.1016/j.cell.2008.09.050.

697 Ramaswami G, Lin W, Piskol R, Tan MH, Davis C, Li JB. Accurate identification of human Alu 698 and non-Alu RNA editing sites. Nature Methods. 2012; 9, 579-581; doi:10.1038/nmeth.1982. 
699 Reenan RA. Molecular determinants and guided evolution of species-specific RNA editing. 700 Nature. 2005; 434, 409-413; doi:10.1038/nature03364.

701 Rieder LE, Staber CJ, Hoopengardner B, Reenan RA. Tertiary structural elements determine the 702 extent and specificity of messenger RNA editing. Nature Communications. 2013; 4; 703 doi:10.1038/ncomms3232.

704 Rousselle M, Simion P, Tilak M-K, Figuet E, Nabholz B, Galtier N. Is adaptation limited by 705 mutation? A timescale-dependent effect of genetic diversity on the adaptive substitution rate in 706 animals. PLOS Genetics. 2020; 16, e1008668; doi:10.1371/journal.pgen.1008668.

707 Savva YA, Rieder LE, Reenan RA. The ADAR protein family. Genome Biology. 2012; 13, 252; 708 doi:10.1186/gb-2012-13-12-252.

709 Smith JM. What Determines the Rate of Evolution? The American Naturalist. 1976; 110(973):3317108.

711 Soldatov RA, Vinogradova SV, Mironov AA. RNASurface: fast and accurate detection of locally 712 optimal potentially structured RNA segments. Bioinformatics. 2013; 30, 457-463; 713 doi:10.1093/bioinformatics/btt701.

714 Tan, MH, Li Q, Shanmugam R, Piskol R, Kohler J, Young AN, Liu KI, Zhang R, Ramaswami G, 715 Ariyoshi K, Gupte A, Keegan LP, George CX, Ramu A, Huang N, Pollina EA, Leeman DS, 716 Rustighi A, Goh YPS, GTEx Consortium; Laboratory, Data Analysis \&Coordinating Center 717 (LDACC)—Analysis Working Group; Statistical Methods groups-Analysis Working Group; 718 Enhancing GTEx (eGTEx) groups; NIH Common Fund; NIH/NCI; NIH/NHGRI; NIH/NIMH; 719 NIH/NIDA; Biospecimen Collection Source Site-NDRI; Biospecimen Collection Source Site720 RPCI; Biospecimen Core Resource_-VARI; Brain Bank Repository_University of Miami Brain 721 Endowment Bank; Leidos Biomedical—Project Management; ELSI Study; Genome Browser Data 
722 Integration \&Visualization-EBI; Genome Browser Data Integration \&Visualization-UCSC

723 Genomics Institute, University of California Santa Cruz; Chawla A, Del Sal G, Peltz G, Brunet A, 724 Conrad DF, Samuel CE, O'Connell MA, Walkley CR, Nishikura K, Li JB. Dynamic landscape 725 and regulation of RNA editing in mammals. Nature. 2017; 550, 249-254; 726 doi:10.1038/nature24041.

727 Waddington CH. Genetic Assimilation of an Acquired Character. Evolution. 1953a; 7, 118-126; 728 doi:10.1111/j.1558-5646.1953.tb00070.x.

729 Waddington CH. The Baldwin Effect, Genetic Assimilation AND Homeostasis. Evolution. 1953b; 730 7, 386-387; doi:10.1111/j.1558-5646.1953.tb00099.x.

731 Wang AH-J, Hakoshima T, van der Marel G, van Boom JH, Rich A. AT base pairs are less stable 732 than GC base pairs in Z-DNA: The crystal structure of d(m5CGTAm5CG). Cell. 1984; 37, 321733 331; doi:10.1016/0092-8674(84)90328-3.

$734 \mathrm{Xu} \mathrm{G}$, Zhang J. Human coding RNA editing is generally nonadaptive. Proceedings of the National 735 Academy of Sciences. 2014; 111, 3769-3774; doi:10.1073/pnas.1321745111.

736 Yablonovitch AL, Deng P, Jacobson D, Li JB. The evolution and adaptation of A-to-I RNA 737 editing. PLOS Genetics. 2017; 13, e1007064; doi:10.1371/journal.pgen.1007064.

738 Yampolsky LY, Stoltzfus A. Bias in the introduction of variation as an orienting factor in 739 evolution. Evolution and Development. 2001; 3, 73-83; doi:10.1046/j.1525740 142x.2001.003002073.x.

741 Yang Y, Lv J, Gui B, Yin H, Wu X, Zhang Y, Jin Y. A-to-I RNA editing alters less-conserved 742 residues of highly conserved coding regions: Implications for dual functions in evolution. RNA. $743 \quad 2008 ; 14,1516-1525 ;$ doi:10.1261/rna.1063708. 
744 Yang Z. PAML 4: Phylogenetic Analysis by Maximum Likelihood. Molecular Biology and

745 Evolution. 2007; 24, 1586-1591; doi:10.1093/molbev/msm088.

746 Yu Y, Zhou H, Kong Y, Pan B, Chen L, Wang H, Hao P, Li X. The Landscape of A-to-I RNA

747 Editome Is Shaped by Both Positive and Purifying Selection. PLOS Genetics. 2016; 12, e1006191;

748 doi:10.1371/journal.pgen.1006191.

749 
Figure 1

Prevalent mRNA editing in coleoid molluscs

(A) Phylogenetic tree of the species taken from TimeTree (Hedges et al. 2006). The asterisk marks the putative beginning of editing site expansion (Liscovitch-Brauer et al. 2017).

Distributions of per-nucleotide editing levels of the predicted editing sites in the studied coleoids.

A

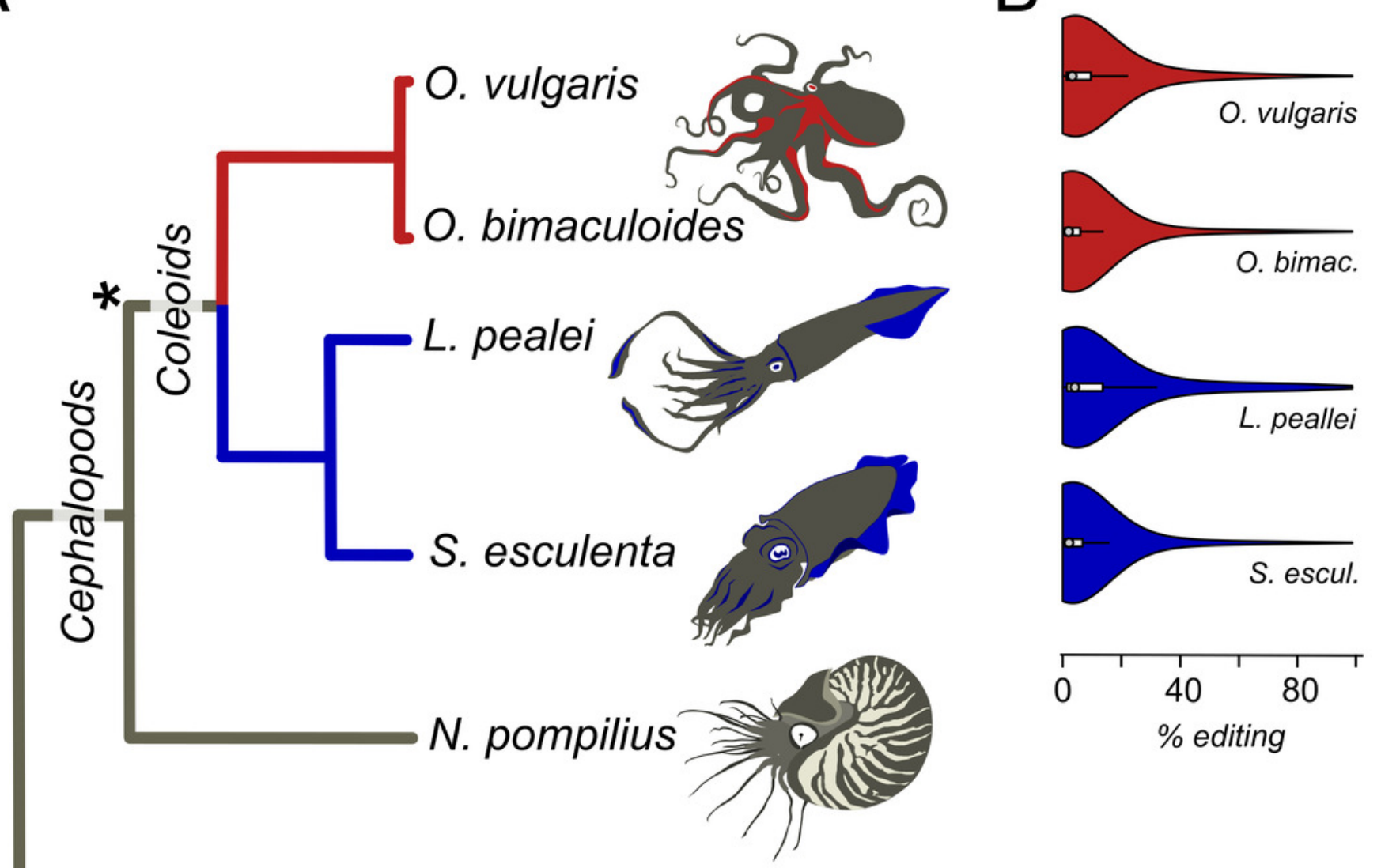

\section{B}

\section{A. californica}

mya 


\section{Figure 2}

Coleoid editing site contexts

(A) O. bimaculoides editing site context changes with the increase of editing level. The height of the letters represents the LOGO bit score of each nucleotide. (B) Highly conserved editing sites tend to be relatively more structured. The fraction of editing sites that are in structured segments is shown for different editing levels: red -0 . vulgaris, red dashed -0 .

bimaculoides, blue $-L$. pealei, blue dashed $-S$. esculenta, grey - the constant showing the fraction of unedited adenines located in structured segments. The noisy pattern at the right is due to a low number of very highly edited sites. (C) The stability of the local secondary structure is higher at edited adenines than at homologous, non-edited adenines for the squid/cuttlefish pair. The distribution of the difference of the minimal free energy Z-score between homologous sites in squid and cuttlefish is shown in blue when two homologous sites have different editing status (edited minus unedited) and in grey when both sites in a pair are edited. The left tail of the blue histogram is heavier than the right one $(p=$

$9.37 \times 10^{-33}$ versus 0.32 for the grey histogram), showing that the editing sites tend to regions with higher secondary structure stability. (D). Conserved editing sites tend to be more structured. The three groups of sites are those present in two of the four species (O. vulgaris and $O$. bimaculoides, red, or L. pealei and S. esculenta, blue), or in all four species (grey). Statistically significant differences are shown with brackets $(* * * p<0.001, * p<0.05)$. 
A

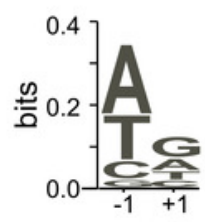

$0-10 \%$

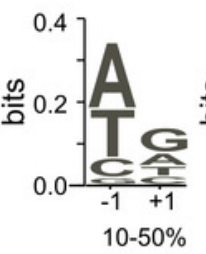

0.2

EL interval

B

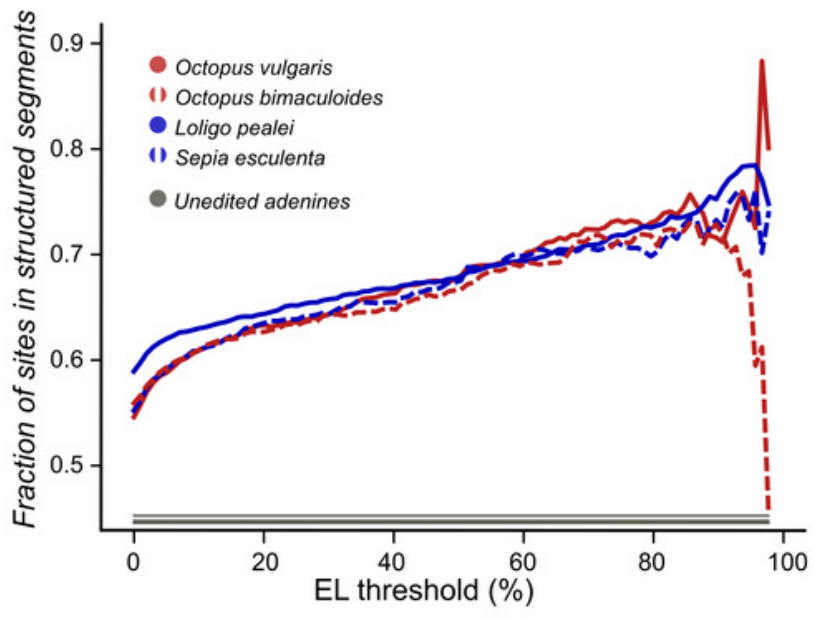

C

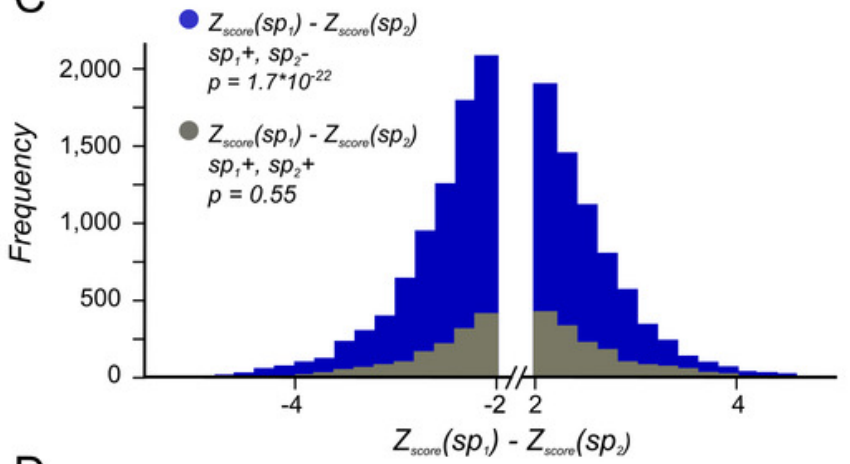

D

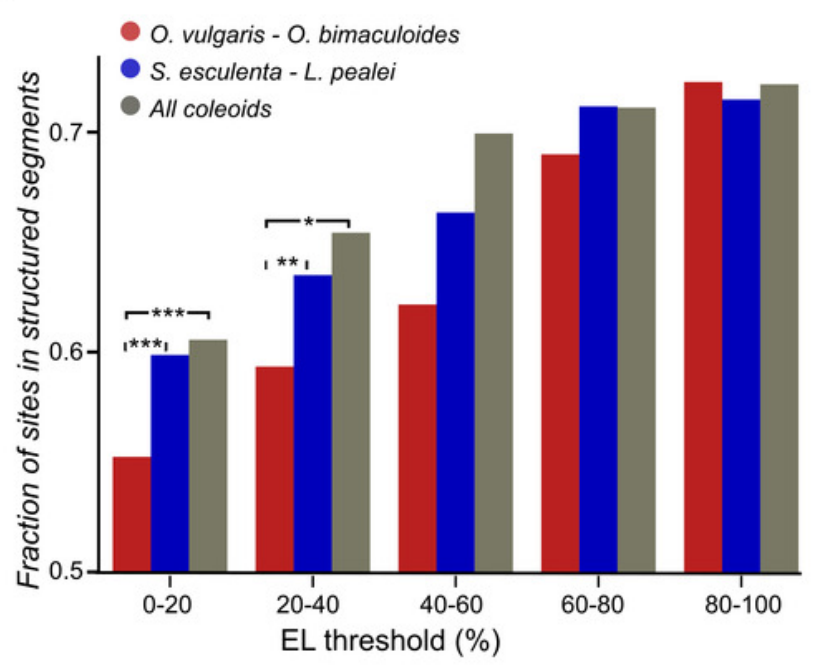


Figure 3

$R$ and $Q$ values.

Dependence of $\boldsymbol{R}_{G}(A)$ and $\boldsymbol{R}_{Y}(B)$ on the editing level. Two curves for each pair are given, since $R_{N}$ is calculated two times for each pair of species using one of them a a reference each time. The red curves correspond to the pair 0 . vulgaris - 0 . bimaculoides; the blue curves, to the pair cuttlefish-squid, the grey curves, to distant pairs. (C). Mutational characteristics of editing sites for the squid-cuttlefish summary substitution matrix. Left to right: $Q_{\rightarrow *}>>1, R_{\rightarrow \mathrm{G}}>1, R_{\rightarrow \mathrm{Y}}<1, Q_{*_{\rightarrow}}>1, R_{\mathrm{G} \rightarrow}>>1, R_{\mathrm{Y} \rightarrow}>1$.
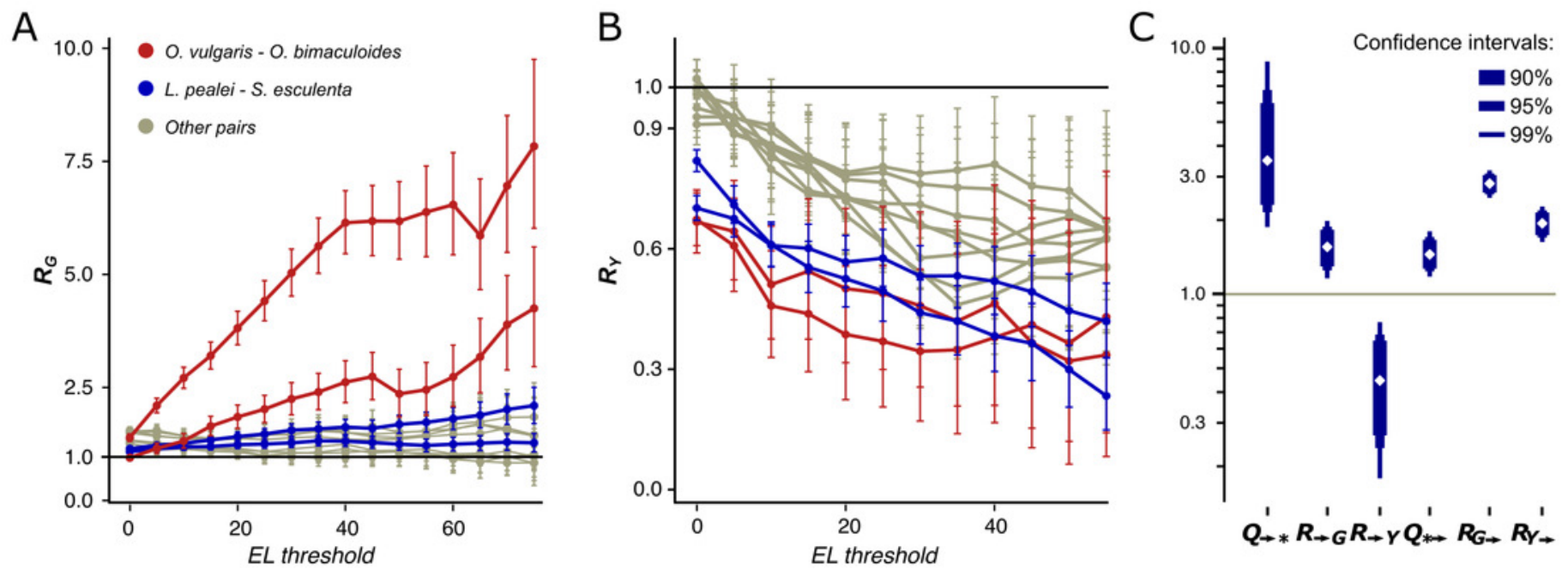
Figure 4

$d N / d S$ values of adenine substitutions to guanines for various EL thresholds

Non-synonymous substitutions are calculated for edited adenines, and synonymous, for unedited adenines. Error bars indicate the $95 \%$ probability value range. A. Plot for the whole range of $d N / d S$ values. B. Truncated value range. Note the increase of $d N / d S$ values at high EL values for all species pairs.

A

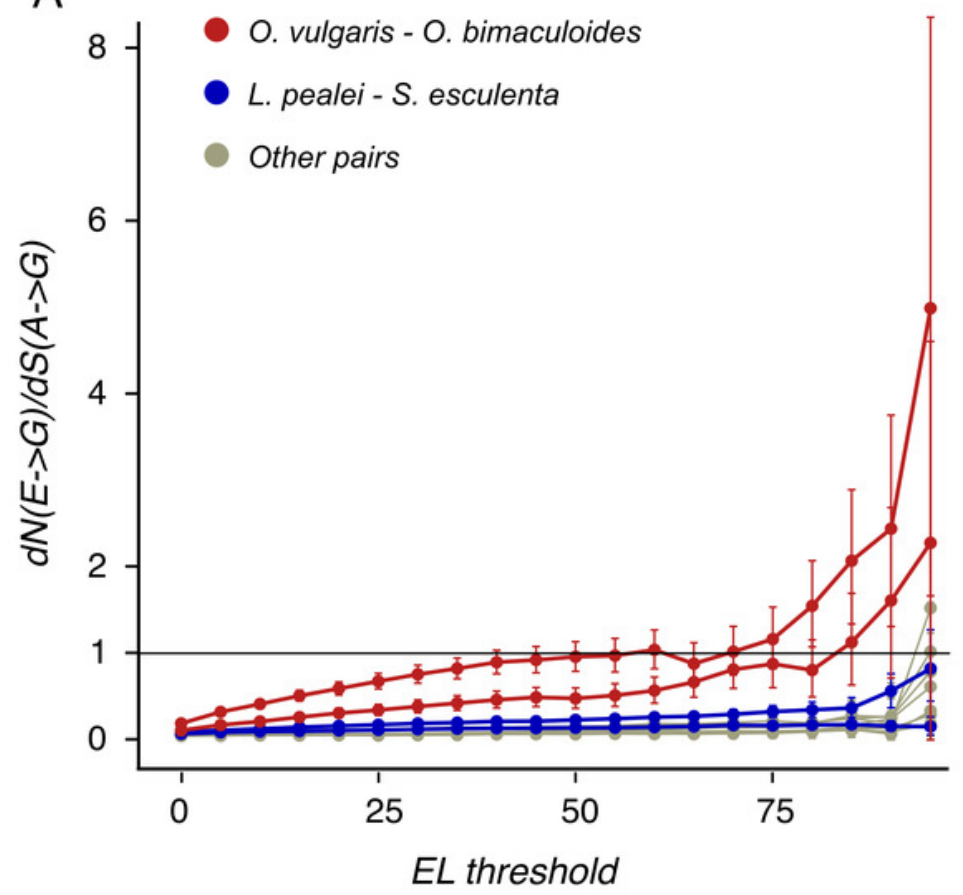

B

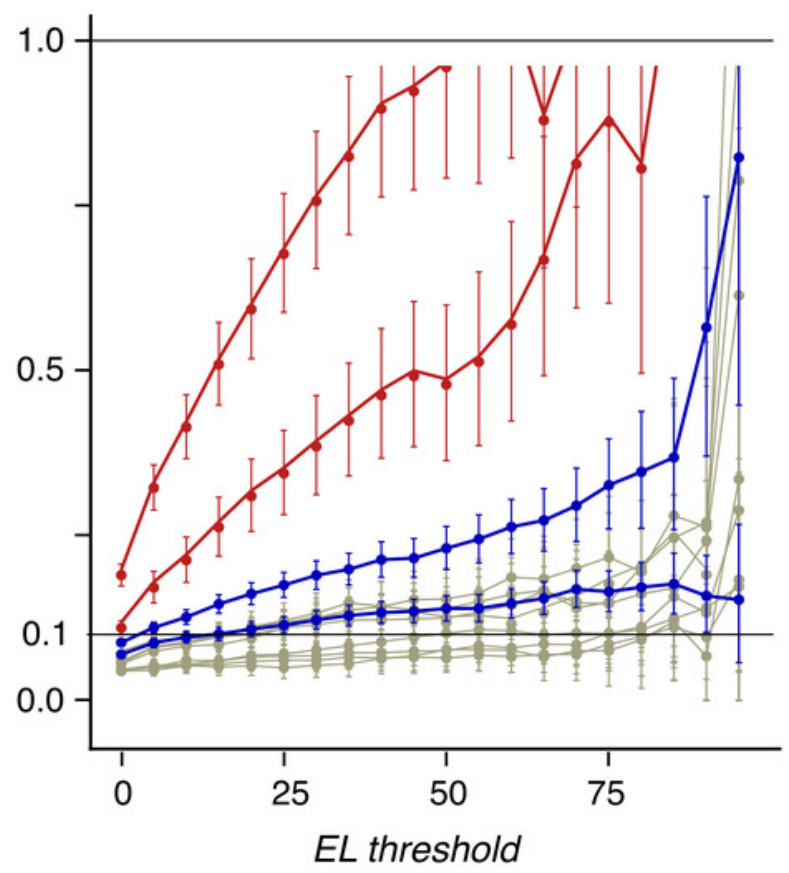

